\title{
An Enhanced Brain Tumor Area Detection and Segmentation Techniques in MRI Medical Images using Modified K-Means Algorithm
}

\author{
Saumya Gupta \\ M. Tech Student \\ Computer Science \& Engineering \\ Oriental Institute of Science \& \\ Technology Bhopal, Madhya \\ Pradesh, India
}

\author{
Monika Agrawal \\ Assistant Professor \\ Computer Science \& Engineering \\ Oriental Institute of Science \& \\ Technology Bhopal, Madhya \\ Pradesh, India
}

\author{
Sanjay Kumar Sharma \\ Head of Department \\ Computer Science \& Engineering \\ Oriental Institute of Science \& \\ Technology Bhopal, Madhya \\ Pradesh, India
}

\begin{abstract}
In medical image processing, one amongst the most important goal of the tumor detection. Within the human body there are different tumors deceases in now days. Brain tumor is very unsafe deceases for human body. Medical image processing provides a good solution for brain tumor detection with the help of MRI tumor image segmentation. Image segmentation refers to the method of partitioning an image into mutually exclusive regions. It may be considered as the most essential and crucial method for facilitating the delineation, characterization, and visualization of regions of interest in any medical image. Despite intensive analysis, segmentation remains a challenging issue due to the various image content, cluttered objects, occlusion, image noise, non-uniform object texture, and other factors. There are many algorithms and techniques accessible for image segmentation but still there has to develop an efficient, fast technique of medical image segmentation. This paper presents an efficient image segmentation methodology using K-means clustering technique.
\end{abstract}

\section{Keywords}

K-mean, MRI images, CT-Scan, KM, EM, FCM

\section{INTRODUCTION}

The MRI scan is more comfortable than CT scan for diagnosis. It is not affect the anatomy because it will not use any radiation. It is based on the magnetic flux and radio waves. There are different types of algorithmic program were developed for brain tumor detection. However they will have some downside in detection and extraction. During this project, 2 algorithms are used for segmentation. Thus it offers the accurate result for tumor segmentation. Tumor is owing to the uncontrolled growth of the tissues in any part of the body. [1] The tumor is also primary or secondary. If it is an origin, then it's referred to as primary. If the part of the neoplasm is spread to a distinct place and grown as its own then it is mentioned as secondary. Normally brain tumor affects CSF (Cerebral Spinal Fluid). It causes for strokes. The physician offers the treatment for the strokes instead of the treatment for tumor. So detection of tumor is very important for that treatment. The lifetime of the person who affected by the brain tumor can increase if it is detected at current stage. That may increase the lifetime about one to two years. [2]

\subsection{Operations Types of Tumor}

In medical imaging, 3D segmentation of images plays a vital role in stages that occur before executing object recognition. 3D image segmentation helps in automated identification of brain diseases and helps in qualitative and quantitative analysis of images like measure accurate size and volume of spotted portion. Accurate measurements in brain diagnosis are quite robust because of numerous shapes, sizes and appearances of tumors. Tumors will grow suddenly inflicting defects in neighboring tissues conjointly, which provides an overall abnormal structure for healthy tissues as well. We will improve a method of 3D segmentation of a brain tumor by using segmentation in combination with morphological operations [8].

\subsection{Tumor}

The word tumor is a synonym for a word tumor that is made by an abnormal growth of cells tumor is something completely different from cancer.

\subsection{Types of Tumor}

There are 3 common kinds of tumor: 1) Benign 2) Pre Malignant 3) Malignant (cancer can solely be malignant) 1) Benign Tumor: A benign neoplasm is a tumor is the one that does not expand in an abrupt way; it doesn't have an effect on its neighboring healthy tissues and together does not expand to non-adjacent tissues. Moles are the common example of benign tumors. 2) Pre-Malignant tumor: Premalignant Tumor is a precancerous stage, considered as a disease, if not properly treated it should cause cancer. 3) Malignant Tumor: Malignancy (mal- = "bad" and -ignis = "fire") is that the sort of growth, that grows worse with the passage of time and ultimately results in the death of an individual. Malignant is basically a medical term that defines a simple progressing disease. Malignant tumor is a term that is usually used for the outline of cancer. [9]

\section{MEDICAL IMAGING TECHNIQUES}

Brain tumors represent a unique challenge in that they that is the organ that is the essence of the "self." moreover, because every area of the brain serves a distinct however important function, the therapy that is most operative for former cancers- surgical removal of either the whole organ or the tumor with a substantial edging of normal tissue - cannot be wont to cure brain tumors. Unfortunately, most brain tumors are relatively insensitive to other cancer treatment, including radiation and chemotherapy. Coupled with the issue in treating brain tumors is the unique biology of the brain.

\subsection{MRI}

Magnetic Resonance Imaging (MRI), Nuclear Magnetic Resonance Imaging (NMRI), or Magnetic Resonance Tomography (MRT) is a medical imaging technique utilized in radiology to envision detailed internal structures. MRI 
makes use of the property of Nuclear Magnetic Resonance (NMR) to image nuclei of atoms inside the body. [4]

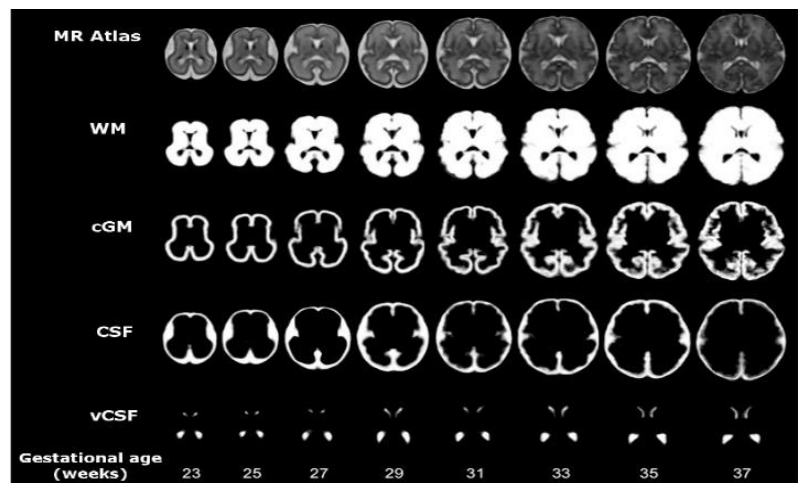

Fig. 1.1: Data Set of MRI image

\subsection{CT SCAN}

A CT scan stands for computed tomography scan. It's conjointly known as a CAT (Computer Axial Tomography) scans. It's a medical imaging methodology that employs tomography. Tomography is the technique of generating a two-dimensional image of a slice or section through a threedimensional object (a tomogram). Interest in computed tomography comes from the fact that, contrary to nuclear medicine methods, it's widely accessible and offers high spatial resolution images with fast acquisition modes: a slice may be acquired in less than a second, with common spatial resolution of regarding $1 \mathrm{~mm}$. Most CT equipment consists of an X-ray tube and an exact number of detecting elements rotating along around the patient, at identical time as the patient table advances though the detection ring, that provides a "spiral" or "helicoidal" acquisition. Contrast in CT images arises from interactions of X-rays with tissues. There are different sorts of interaction, the principal one being the photoelectric impact.

\section{PROPOSED METHOD}

The clustering of numerical data forms the premise of many classification and system modeling algorithms. The aim of clustering is to identify natural groupings of data from a large data set to provide a concise illustration of a system's behavior. K-means clustering is a methodology of vector quantization, originally from signal processing, that is common for cluster analysis in data mining. K-means clustering aims to partition $\mathrm{n}$ observations into $\mathrm{k}$ clusters among which each observation belongs to the cluster with the closest mean, serving as a model of the cluster. This finishes up during a partitioning of the data space into Voronoi cells.

The algorithmic rule includes a loose relationship to the knearest neighbor classifier, a standard machine learning technique for classification that is often confused with kmeans because of the $\mathrm{k}$ among the name. One can apply the 1nearest neighbor classifier on the cluster centers obtained by $\mathrm{k}$-means to classify new data into the prevailing clusters. This is referred to as nearest centroid classifier or Rocchio algorithm.

\subsection{K-means Clustering Algorithm}

Clustering could be a kind of unsupervised learning of grouping together similar data items into one cluster consistent with some selection criteria. Clustering is a main task of explorative data mining, and a standard technique for statistical data analysis utilized in many fields, together with machine learning, pattern recognition, image analysis, data retrieval, and bioinformatics. Differing kinds of algorithms have been designed based on clustering model like connectivity, centroid, distribution models etc. clustering is a methodology of data exploration that extracts the patterns from images according to interest.

$\mathrm{K}$-means is one of the simplest unsupervised learning algorithms that solve the renowned clustering drawback. The procedure follows an easy and simple way to classify a given data set through an explicit range of clusters (assume $\mathrm{k}$ clusters) fixed apriori. The main idea is to outline $\mathrm{k}$ centers, one for every cluster. These centers ought to be placed during a cunning manner because of different location causes different outcome. So, the better option is to put them as much as possible distant from each other. The next step is to require every point belonging to a given data set and associate it to the nearest center. When no point is pending, the primary step is completed and an early cluster age is completed. At this time we have to re-calculate k new centroids as barycenter of the clusters resultant from the previous step. When we have these $\mathrm{k}$ new centroids, a new binding has to be done between identical data set points and the nearest new center. A loop has been generated. As a result of this loop we tend to may notice that the $\mathrm{k}$ centers change their location step by step till no additional changes are done or in alternative words centers do not move any more.

K-Means algorithm has many benefits like:

a. There are invariably $\mathrm{K}$ clusters.

b. There is invariably at least one item in every cluster.

c. The clusters are non-hierarchical and they do not overlap.

d. Every member of a cluster is nearer to its cluster than the opposite cluster because closeness does not invariably involve the 'center' of clusters.

e. If variables are large, then K-Means most of the times computationally faster than hierarchical clustering, if we have $\mathrm{k}$ smalls.

f. K-Means generate tighter clusters than hierarchical clustering, specifically if the clusters are globular.

\subsection{Mathematical Representation}

Finally, this algorithm objects at minimizing an objective function know as squared error function specified by:

$$
J(V)=\sum_{i=1}^{C} \sum_{j=1}^{C}\left(\left\|x_{i}-v_{j}\right\|\right)^{2}
$$

Where

' ||$x_{i}-v_{j} \mid l$ ' is the Euclidean distance between $x_{i}$ and $v_{j}$.

' $c_{i}$ ' is the number of data points in $i^{\text {th }}$ cluster.

' $c$ ' is the number of cluster centers.

\subsection{Algorithmic Steps for K-Means Clustering}

Let $X=$ be the set of data points and $V=$ be the set of centers.

1. Randomly select ' $c$ ' cluster centers.

2. Calculate the area between every data point and cluster centers. 
3. Assign the data point to the cluster center whose distance from the cluster center is minimum of all the cluster centers.

4. Re-calculate the new cluster center using:

\begin{tabular}{|l|l|}
\hline$v_{i}=\left(1 / c_{i}\right) \sum_{j=1}^{c_{i}} x_{i}$ & (2) \\
\hline
\end{tabular}

where, ' $c c_{i}$ ' represents the number of data points in $i^{\text {th }}$ cluster.

5. Re-calculate the area between every data point and new obtained cluster centers.

6. If no data point was reassigned then stop, otherwise repeat from step 3).

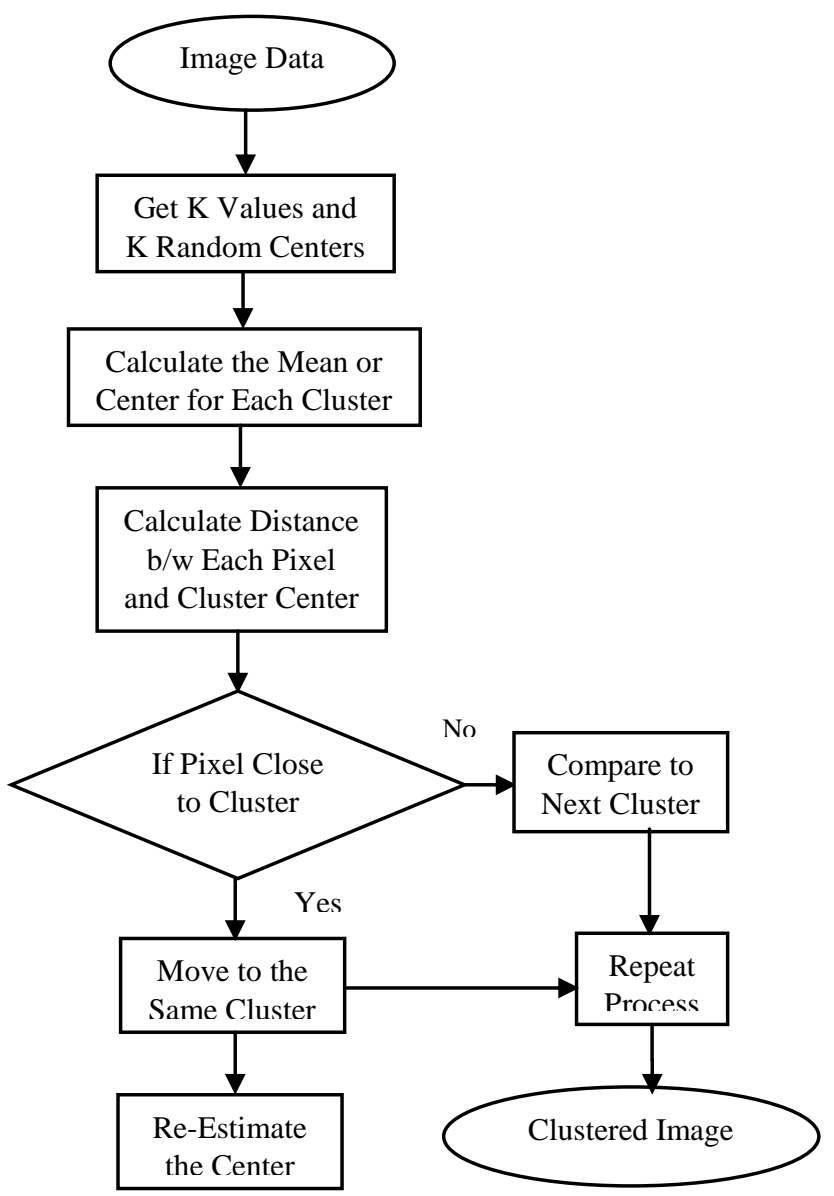

Fig. 3.1: Flowchart of proposed K-mean algorithm

\subsection{Steps for Proposed Improved K-Mean} Algorithm

1. Give the no of cluster value as $\mathrm{k}$.

2. Randomly choose the k cluster centers.

3. Calculate mean or center of the cluster.

4. Calculate the distance $\mathrm{b} / \mathrm{w}$ each pixel to each cluster center.

5. If the distance is near to the center then move to that cluster.

6. Otherwise move to next cluster

7. Re-estimate the center.
8. Repeat the process until the center doesn't move.

9. Apply morphological operation to improve the accuracy of the output.

10. Finally got the clustered output of the image.

11. Select the better cluster of the output of the image.

12. Final output of the cluster in shown in next figure window.

13. There are two figure in this window shows one shows the cluster output image.

14. For detecting the quality of cluster image employing "Canny Edge detection" - shows the accurateness of the proposed method.

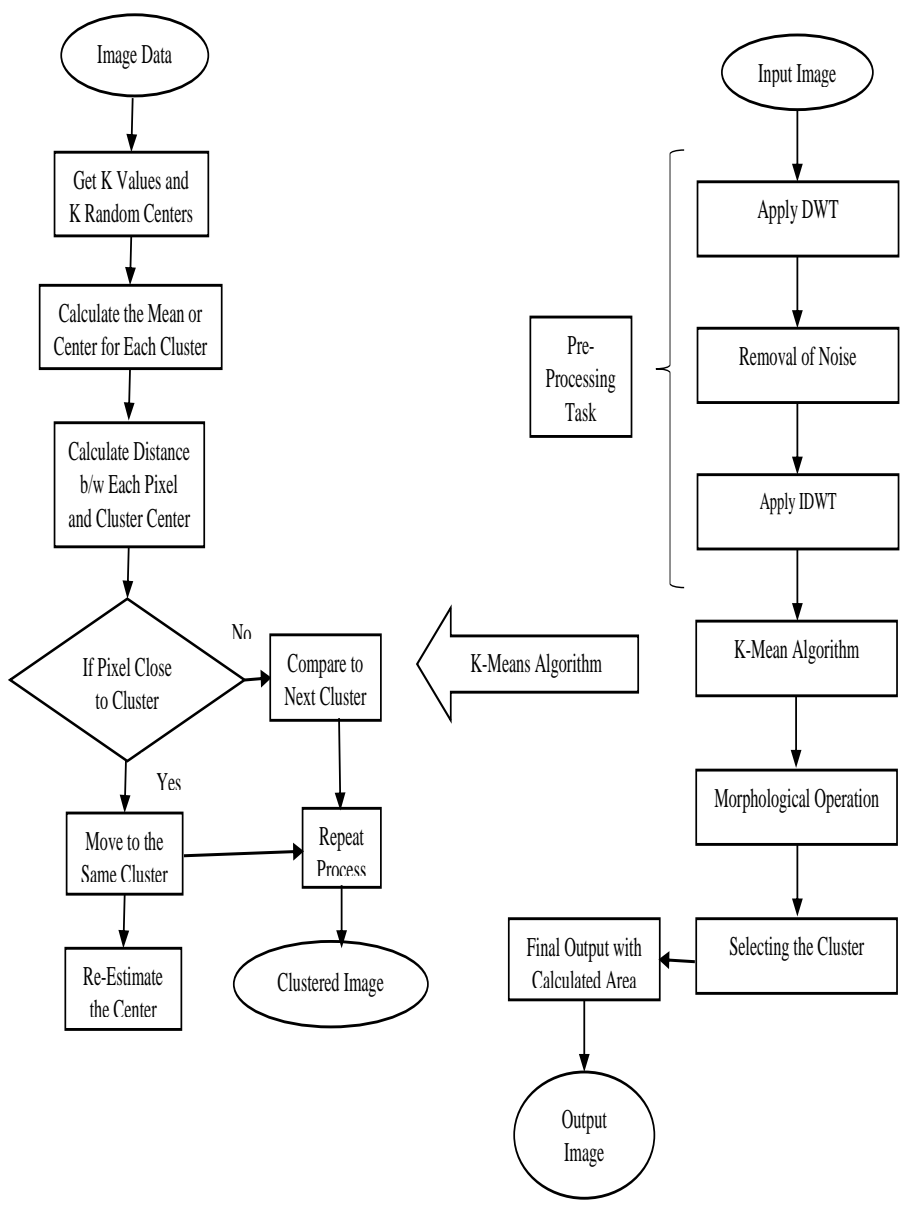

Fig. 3.2: Flowchart of proposed improved K-mean algorithm

\section{SIMULATION AND RESULT}

In this section, we tend to show the results of our projected image segmentation technique that obtained using real MRI brain images from 3 different data sets. This work was implemented using MATLAB 7.12.0 (R2011a). We tend to run our experiments on a core $15 / 2.4 \mathrm{GHz}$ computer with 4 GB RAM and an NVEDIA/ (1 GB VRAM) VGA card. [10] So as to examine the performance of our image segmentation approach, we tend to used 3 benchmark data sets. The primary one is that the Digital Imaging and Communications in medicine (DICOM) data set. DICOM consists of 22 images that contain brain tumors. All DICOM image files are encoded in JPEG2000 transfer syntax with "DCM" extension. It has no ground truth images for the contained images. The 
second data set is Brain web data set. It contains simulated brain MRI data based on 2 anatomical models: normal and multiple sclerosis (MS). Full 3-dimensional data volumes are simulated using 3 sequences (T1-, T2-, and proton density(PD-) weighted) and a range of slice thicknesses, noise levels, and levels of intensity non-uniformity. The files contained during this data set have extension of "MNC".

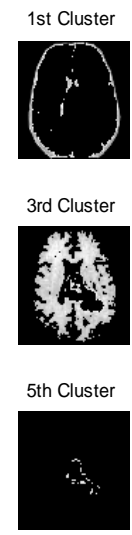

Fig. 4.1: Proposed Output of data set (DS)-1 of all five cluster

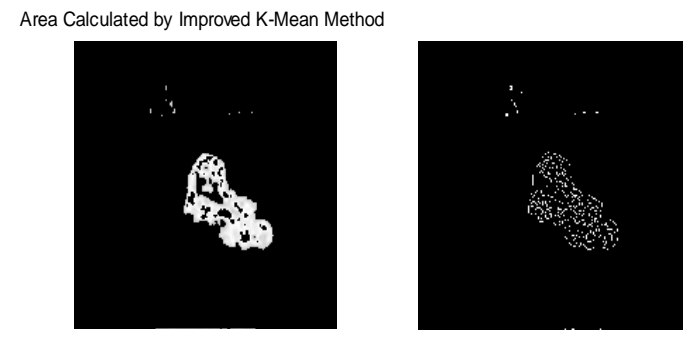

Fig. 4.2: Proposed Output of data set (DS)-1 of cluster-4
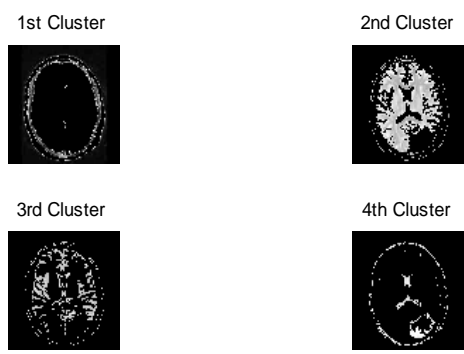

5th Cluster

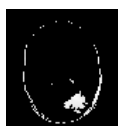

Fig.4.3: Proposed Output of data set (DS)-3 of all five cluster

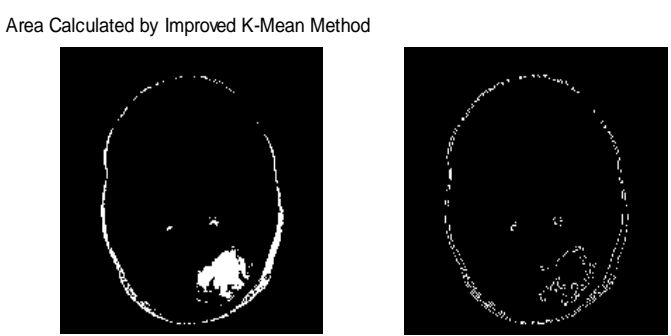

Fig.4.4: Proposed Output of data set (DS)-3 of cluster 5
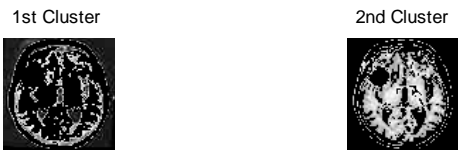

3rd Cluster

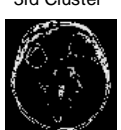

4th Cluster

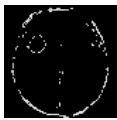

5th Cluster

$\checkmark$

Fig.4.5: Proposed Output of data set (DS)-4 of all five cluster

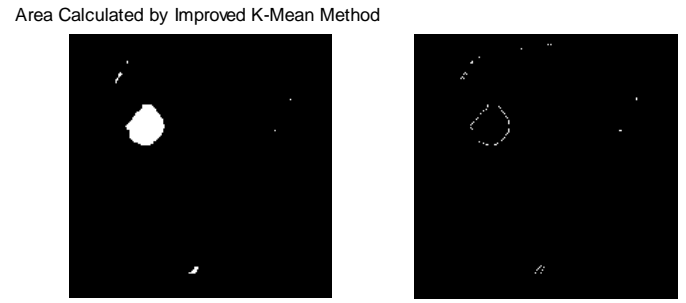

Fig. 4.6: Proposed Output of data set (DS)-4 of cluster 5

Table 4.1: Timing Comparison of different methods

\begin{tabular}{|c|c|c|c|}
\hline Method & DS 1 & DS 3 & DS 4 \\
\hline KM & 7.52 & 1.76 & 4.34 \\
\hline EM & 34.47 & 8.00 & 32.06 \\
\hline FCM & 59.52 & 15.92 & 6.89 \\
\hline KIFCM & 12.87 & 5.18 & 3.46 \\
\hline $\begin{array}{c}\text { Proposed } \\
\text { Method }\end{array}$ & 3.35 & 3.51 & 3.39 \\
\hline
\end{tabular}

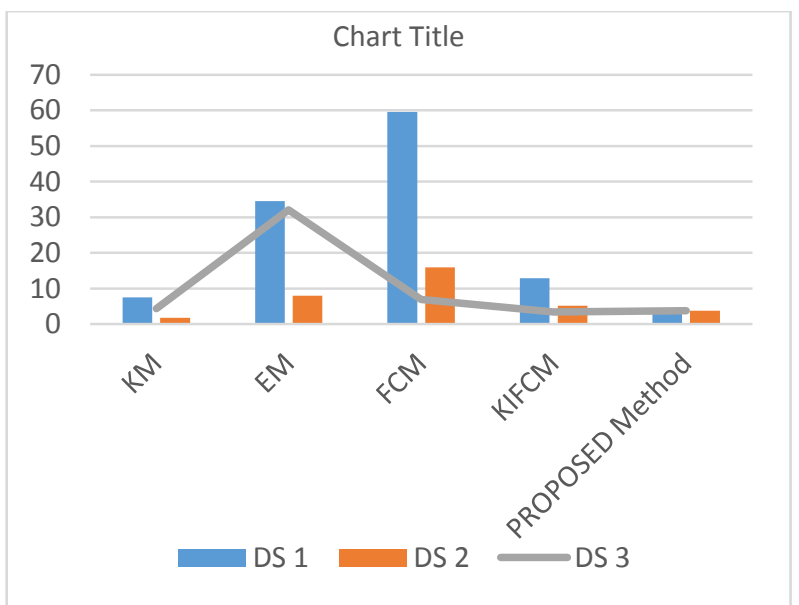

Fig. 4.7: Graphical representation of time comparison

Figure 4.7 shows the graphical representation of timing comparison of different methods. There are different methods like KM, EM, FCM, KIFCM and our proposed method. Results shows that our proposed method have improved timing results than other methods. 


\section{CONCLUSION}

There are different types of tumors are available. They may be as mass in brain or malignant over the brain. Suppose if it is a mass then $\mathrm{K}$ - means algorithm is enough to remove it from the brain cells. If there is any noise are present in the MRI image it is removed before the $\mathrm{K}$-means process. The noise free image is assumed as an input to the k-means and tumor is removed from the MRI image. The experimental results are compared with other algorithms. The final output of enhanced $\mathrm{K}$-mean calculated area with improved accuracy is shown in the proposed work. The accuracy is observed with the help of Edges of proposed $\mathrm{K}-$ Mean algorithm.

\section{REFERENCES}

[1] J. Selvakumar, A. Lakshmi, T. Arivoli, "Brain Tumor Segmentation and Its Area Calculation in Brain MR Images using K-Mean Clustering and Fuzzy C-Mean Algorithm”, IEEE-International Conference On Advances In Engineering, Science And Management (ICAESM -2012) 2012, pp-186-190.

[2] Phooi Yee Lau, Frank C. T. Voon, and Shinji Ozawa," The detection and visualization of brain tumors on T2weighted MRI images using multi-parameter feature blocks", Proceedings of the 2005 IEEE Engineering in Medicine and Biology 27th Annual Conference Shanghai, China 2005, pp-5104-5107.

[3] Dipali M. Joshi, Dr. N. K. Rana and V. M. Mishra, "Classification of Brain Cancer Using Artificial Neural Network", International Conference on Electronic Computer Technology 2010, pp-112-116

[4] Carlos Arizmendi, Juan Hernández-Tamames, Enrique Romero, Alfredo Vellido, Francisco del Pozo, "Diagnosis of Brain Tumors from Magnetic Resonance Spectroscopy using Wavelets and Neural Networks", Annual International Conference of the IEEE EMBS 2010, pp-6074-6077

[5] Arpita Das, Mahua Bhattacharya, "A Study on Prognosis of Brain Tumors Using Fuzzy Logic and Genetic Algorithm Based Techniques", International Joint Conference on Bioinformatics, Systems Biology and Intelligent Computing 2009, pp-348-351
[6] Ahmed Kharrat, Mohamed Ben Messaoud, "Detection of Brain Tumor in Medical Images", International Conference on Signals, Circuits and Systems 2009, pp-16

[7] Jason J. Corso, Eitan Sharon, Shishir Dube, Usha Sinha, and Alan Yuille, "Efficient Multilevel Brain Tumor Segmentation With Integrated Bayesian Model Classification”, IEEE TRANSACTIONS ON MEDICAL IMAGING, VOL. 27, NO. 5, MAY 2008, pp-629-640

[8] Hassan Khotanlou, Olivier Colliot, Jamal Atif, Isabelle Bloch, "3D brain tumor segmentation in MRI using fuzzy classification, symmetry analysis and spatially constrained deformable models", Elsevier, Fuzzy Sets and Systems 160 (2009) 1457-1473

[9] M. Masroor Ahmed, Dzulkifli Bin Mohamad, "Segmentation of Brain MR Images for Tumor Extraction by Combining K-means Clustering and Perona-Malik Anisotropic Diffusion Model", International Journal of Image Processing, Volume (2) : Issue(1), pp-27-34

[10] Pranay Yadav, "Color Image Noise Removal by Modified Adaptive Threshold Median Filter for RVIN", Electronic Design, Computer Networks \& Automated Verification (EDCAV), 2015 International Conference on National Institution of Technology (NIT - Shilog) Conference, pp - 175 - 180, 29-30, DOI, 10.1109/EDCAV.2015.7060562, Jan. 2015

[11] Prany Yadav1 and Parool Singh2, "Color Impulse Noise Removal by Modified Alpha Trimmed Median Mean Filter for FVIN", IEEE International Conference on Computational Intelligence and Computing (IEEEICCIC) in PARK College of Engineering and Tekhnology, Coimbatore-641659, pp: $1-8$, DOI: 10.1109/ICCIC.2014.7238369, Dec - 2014.

[12] Sharma, S. and Yadav, P, "Removal of Fixed Valued Impulse Noise by Improved Trimmed Mean Median Filter" IEEE International Conference on Computational Intelligence and Computing (IEEE-ICCIC) in PARK College of Engineering and Tekhnology, Coimbatore641659, Tamilnadu, (IEEE-ICCIC), pp: 1 8, DOI: 10.1109/ICCIC.2014.7238368, Dec 2014. 